\title{
Home-based, early rehabilitation after stroke: the perspectives of people with aphasia
} and healthcare professionals

\author{
Dr. Ciara Shiggins ${ }^{1}$ and Dr. Simon Horton ${ }^{1}$ \\ ${ }^{1}$ School of Health Sciences, University of East Anglia, Norwich, UK \\ Corresponding email: c.shiggins@uea.ac.uk
}

\section{Background}

Currently, people with aphasia (PWA) in the UK receive stroke rehabilitation in a variety of care settings, including Early Supported Discharge (ESD) (home based rehabilitation). Stroke survivors, including PWA, may be discharged from in-patient to ESD services, within a day of having a stroke, if the person meets the functional, cognitive and social criteria. Core therapy for PWA includes physiotherapy (PT), occupational therapy (OT) and speech \& language therapy (SLT). Therapy programmes, while devised by qualified professionals, are generally carried out by rehabilitation assistants (RAs) or assistant practitioners (APs), who may not necessarily receive specific training in stroke or aphasia. (Re)learning is key to the success of aphasia rehabilitation (Hopper \& Holland, 2005), with practice being one of the most important learning processes, enhancing neuroplasticity and reducing learned non-use (Kleim, 2011). Despite this, in in-patient acute settings, PWA communicate with healthcare professionals (HCPs) for less than $50 \%$ of their time (Knight, Worrall, \& Rose, 2006). Indeed, early after their stroke, people have been reported to spend $60 \%$ of their time alone (Bernhardt, Dewey, Thrift \& Donnan, 2004), with PWA spending more time alone than people post-stroke without aphasia (Godecke, Armstrong, Hersh \& Bernhardt, 2013). No studies have explored interactions between PWA and their HCPs in ESD services, and little is known about the PWA's or HCPs' experiences and perspectives in this context.

\section{Aims}

To explore experiences of ESD rehabilitation in day-to-day practice from the perspective of HCPs and PWA.

\section{Methods}

This abstract focuses on interview data from a larger study that used both observational and interview methods. Ten PWA and $22 \mathrm{HCPs}$ were recruited to the larger study, including a wide range of professional groups, including: OT; PT; RA; AP; nurses; and PWA with a broad 
range of severity and type of aphasia. On completion of the observational data collection, semi-structured, topic-guided interviews were conducted with a purposive sample of HCP participants $(n=8)$, representing the different HCPs working in the ESD team; years of experience working in stroke rehabilitation and with PWA; and who were still working in the service at the end of the study; and all PWA who completed the study $(n=9)$. Purposive sampling was used in order to obtain a diverse data-rich sample. The semi-structured topic guides explored HCP's and PWA's experiences and understanding of stroke and aphasia, and home-based rehabilitation; impacts of aphasia on rehabilitation (HCPs); and communication with rehabilitation team members (PWA). These topic guides were further supplemented and personalised using information gained through observational data. Vignettes from observational data were used as appropriate to contextualise interview questions and support communication. Interview data were analysed using thematic analysis; interview and observation data were analysed and combined within an Activity-based Communication Analysis (ACA) approach.

\section{Findings}

Analysis of HCP and PWA data produced four common themes relating to the research questions: HCP \& PWA emotions; rapport development; use of rehabilitation materials; and the home environment. Three additional themes were particular to HCP data: HCP and PWA collaboration; processes of rehabilitation; and staff training; and two particular to PWA data: journey of aphasia; and PWA's attitudes. Themes were interrelated, conveying opinions and perceptions of routine rehabilitation, within the home context. PWA and HCPs identified both facilitators of and barriers to engagement and interaction in rehabilitation. In addition, the perspectives of PWA and HCPs provided insights and ways to overcome the barriers to (re)learning and opportunities that could be capitalised on to maximise rehabilitation. There were points of congruence and disagreement between the perspectives of PWA and HCPs.

\section{Discussion}

This small sample produced novel insights into the opportunities to enhance (re)learning, particularly communication or conversational practice, which were inconsistently realised. 
This research provided a deeper understanding of the complexities of routine ESD rehabilitation.

\section{Conclusion}

Better understanding of the experience of routine rehabilitation, in the context of ESD, from the perspective of PWA and HCPs, provides us with insights to enhance (re)learning during routine rehabilitation. These findings have clinical implications and warrant further research.

\section{References}

Hopper, T. and Holland, A. L. 2005. Aphasia and learning in adults: Key concepts and clinical considerations. Topics in Geriatric Rehabilitation, 21(4), pp.315-322.

Bernhardt, J., Dewey, H., Thrift, A. and Donnan, G. 2004. Inactive and alone: Physical activity within the first 14 days of acute stroke unit care. Stroke, 35, pp.1005-1009.

Knight, K., Worrall, L. and Rose, T. 2006. The provision of health information to stroke patients within an acute hospital setting: What actually happens and how do patients feel about it? Topics in Stroke Rehabilitation, 13(1), pp.78-97.

Kleim, J. A. 2011. Neural plasticity and neurorehabilitation: Teaching the new brain old tricks. Journal of Communication Disorders, 44(5), pp. 521-528.

Godecke, E., Armstrong, E., Hersh, D. and Bernhardt, J. 2013. Learned communicative nonuse is a reality in very early aphasia recovery: Preliminary results from an ongoing observational study. In Proceedings of the 43rd Clinical aphasiology conference, Tucson, AZ: May 28-June 2, 2013, University of Pittsburg. Pearson 